\title{
TWO-TIER MODEL FOR NUCLEATE POOL BOILING ON MICROCONFIGURED COMPOSITE SURFACES
}

\author{
Nengli Zhang, Wen-Jei Yang and G. W. Yang \\ Department of Mechanical Engineering and Applied Mechanics \\ University of Michigan, Ann Arbor, Michigan 48109
}

(Communicated by J.P. Hartnett and W.J. Minkowycz)

\begin{abstract}
A new model is developed to describe the heat transfer mechanism in nucleate pool boiling on a microconfigured composite surface. Both the microlayer and macrolayer thicknesses are determined from the model. This model can be extended to explain the nucleate boiling on plain surfaces. The enhancement mechanisms of heat transfer for the nucleate boiling on the microconfigured surface are analyzed.
\end{abstract}

\section{Introduction}

Enhanced boiling tubes can achieve up to 10-15 times the nominal surface heat flux of plain tubes. Such enhanced nucleate boiling surfaces have been widely used in industry, but the heat transfer mechanisms of boiling enhancement on the surfaces are still not well understood. Based upon results obtained from the visualization and the fundamental studies performed in the U.S.A., Europe, the former U.S.S.R., and Japan, Thome [1] concluded that enhanced nucleation, thin film evaporation and two phase convection in the reentrant channels were the primary modes for augmenting heat transfer. Gebhart and Wright [2] obtained an increase in the heat dissipation rate at a given liquid superheat on microconfigured silicon surfaces of about four times. Such an enhancement was attributed to the function of high frequency microbubbles [3] whose existence was never detected [4]. Nevertheless, Wright and Gebhart [5] proposed a two bubble model to account for the boiling heat transfer enhancement from microconfigured surfaces. Their model was based upon the postulation that there exist microbubbles in the microlayers under macrobubbles of 0 (1) $\mathrm{mm}$ in diameter. Yang et al. [6] disclosed nucleate pool boiling heat transfer coefficients on a graphite-copper composite surface to be three to six times higher than those on a pure copper surface. The mechanism of such an enhancement was left unexplained. 
In the present paper, a two-tier-mushroom nucleate boiling model is proposed to describe the enhancement mechanisms of nucleate pool boiling on microconfigured composite surfaces.

\section{Mechanism of Nucleate Boiling on Plain Surfaces}

The mechanism of nucleate boiling has, for many decades, attracted considerable attentions among heat transfer researchers. A photographic study on nucleate boiling by Gaertner [7] led to the classification of four distinct regions in the nucleate boiling regime, based on the bubble and vapor morphology, as shown in Fig. 1. In the partial nucleate boiling region, active nucleate sites produce discrete bubbles. As the heat flux is increased, these discrete bubbles begin to disappear, and are replaced by vapor columns and mushrooms, constituting a first transition region. In the vapor mushroom region at a higher heat flux, the heat transfer surface is covered with huge billowing vapor mushrooms. The second transition region appears near the critical heat flux (CHF). It is characterized by the formation of vapor patches on some local arcas of the surface which have a critical active site population. A liquid-rich layer was found to prevail on the boiling surface even when the entire surface was covered with bubbles 18 12]. Yu and Mesler [13] referred to the liquid-rich layer as a macrolayer in order to distinguish it from a microlayer formed within a macrobubble (called an individual bubble) which grows on the heating surface during nucleate boiling. Tiny vapor stems were detected existing inside a macrolayer. Their average length has been treated as macrolayer thickness. The general consensus is that this macrolayer controls the nucleate boiling process at higher heat fluxes, while a microlayer characterizes the partial boiling process -- discrete bubble region. Although several mechanisms were suggested for heat transfer in both the fully established and partial nucleate boiling regions, no satisfactory model can yet explain the processes in the two regions and nor explain the smooth and continuous transition of boiling mechanisms between the two regions [14].

A variety of physical models have been proposed to describe the heat transfer processes in the partial nucleate boiling region, including bubble agitation, micro-convection, vapor-liquid exchange, latent heat transport, transition conduction, microlayer evaporation and others. Since no single model can explain such a complex nucleate boiling process, several mechanisms are considered functioning simultaneously with different weights depending upon each specific operating condition [14]. In the higher flux region, vapor mushrooms are formed from coalesced bubbles with the heat transfer mechanism being different from that of the partial nucleate boiling. The formation of a macrolayer and its evaporation are of major importance in the understanding of the heat transfer process in the higher heat flux region. 

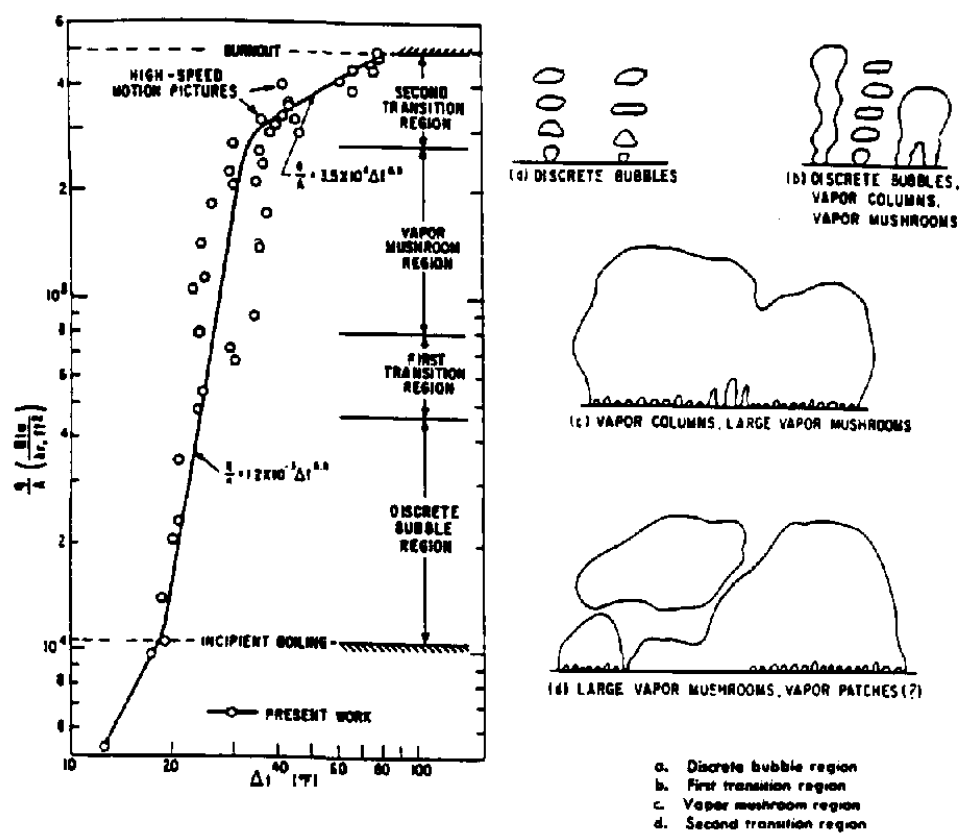

FIG. 1

Heat Transfer Data for Water Boiling on Horizontal Copper Surface and Corresponding Flow Patterns [7]

\section{Microconfigurations on a Graphite-Copper Composite Surface}

The graphite-copper composite material used in reference [6] consists of graphite fibers of $8-10 \mu \mathrm{m}$ diameter imbedded uniaxially within a copper matrix ( $50 \%$ area fraction). Figure 2 shows the photomicrographs of the surface. The thermal conductivity of the graphite fiber is three times higher than that of the base matrix. Each fiber functions like a highly efficient pin fin which is imbedded in the heat transfer surface material. The heat transfer coefficients for nucleate pool boiling on the composite surface are found to be three to six times higher than those on a pure copper surface. Recently, a study [15] was conducted to determine the effects of microconfigurations on the composite surface which caused the measured enhancement. It was revealed that the mean square roughness of the composite surface was about five times that of pure copper when both surfaces were polished using the same diamond abrasive. Each fiber tip on the composite surface appears as a plateau with rugged terrain, while the base material surface (the space between the plateaus) is filled with a large number of microsized low-lying trenches and intermingled narrow grooves, as shown in Fig. 3.

\section{Two-Tier Model}

The nucleate pool boiling performance characteristics are divided into two regions: low and high heat flux regions. 


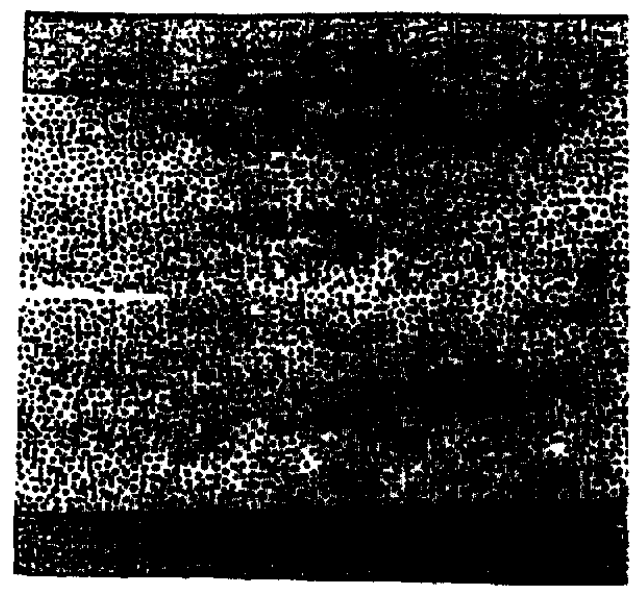

100x Magnifleation

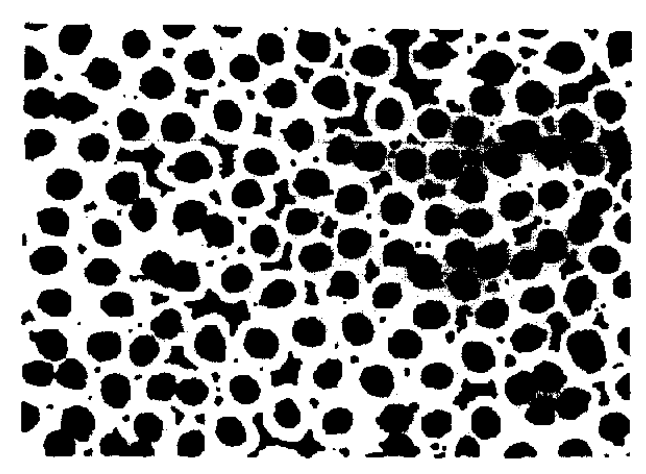

500x Magnification

FIG. 2

Photomicrographs of the Surface on a Graphite Fiber Reinforced Copper Composite Produced by SPARTA

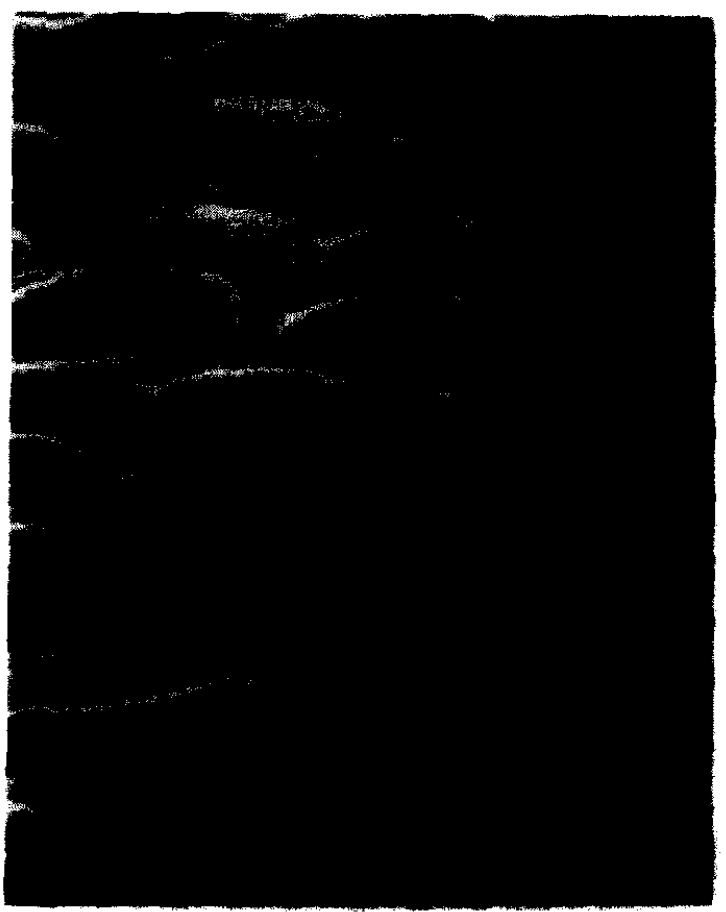

FIG. 3

A Photo of Microconfiguration on Gr-Cu Composite Surface 


\section{(i) Low heat flux region with single-tier configuration}

It is well known that in low heat flux, as in nucleate boiling on a plain surface, discrete vapor bubbles are generated at active nucleation sites and grow due to the vaporization of both the superheated liquid surrounding the bubbles and the thin film in the microlayer trapped beneath the bubbles. However, in the case of boiling on a composite surface, the spaces (on the base matrix surface) between the graphite fiber tips may function as the uniformly distributed nucleation sites and some of these spaces may produce microbubbles of $0(10) \mu \mathrm{m}$ in diameter, which is the same order of magnitude as the spaces themselves. These microbubbles may coalesce and grow with a continuous supply of vapor from the microbubbles to form a small mushroom (or discrete bubble). Each small mushroom is supported by a multiple of micro vapor stems (in columns) which are formed by high-frequency microbubbles generated over the base matrix surface. These micro vapor stems, together with the micro liquid volume being trapped between them, constitute a mixed-phase microlayer, as depicted in Fig. 4. Meanwhile, condensation may occur on the discrete bubble surface which is at a distance above the superheated thermal layer. Generally, one observes a rather uniform distribution of discrete bubbles on the composite surface in the low heat flux nucleate boiling region. This is because the spaces functioning as the nucleation sites are macroscopically, uniformly distributed, but are microscopically, non-uniformly distributed on the base matrix surface, as shown in Fig. 2. The discrete bubbles are generated only on those spaces which meet the nucleating conditions (for example, temperature and morphology). Meanwhile, a great number of microbubbles may detach from the individual active sites without coalescing because of the large distance between them. These quickly condense immediately after their departure from the heating surface. The microbubbles which form the micro vapor stems and those which condense upon the departure constitute the major contributors to the measured enhancement. Figure 4 illustrates a single-tier configuration in the partial nucleate boiling region, i.e. low heat flux boiling, on a composite surface.

\section{(ii) High heat flux region with two-tier configuration}

As the heat flux is raised, the number of discrete bubbles increases and some groups of adjacent bubbles coalesce at their widest cross sections to form larger vapor masses, called macro mushrooms. Each macro mushroom is supported by a macro layer which consists of macro vapor stems and macro liquid volumes that are trapped between them. The macro vapor stems are formed by the discrete bubbles, each of which is suspended on one microlayer. If each macro vapor stem is regarded as a small mushroom, then each mushroom - together with a microlayer underneath it - is equivalent to a single-tier configuration shown in Fig. 4. Furthermore, the macro mushroom, together with the macrolayer underneath it, constitutes another tier configuration. In other words, the system illustrated in Fig. 5 is a two-tier 


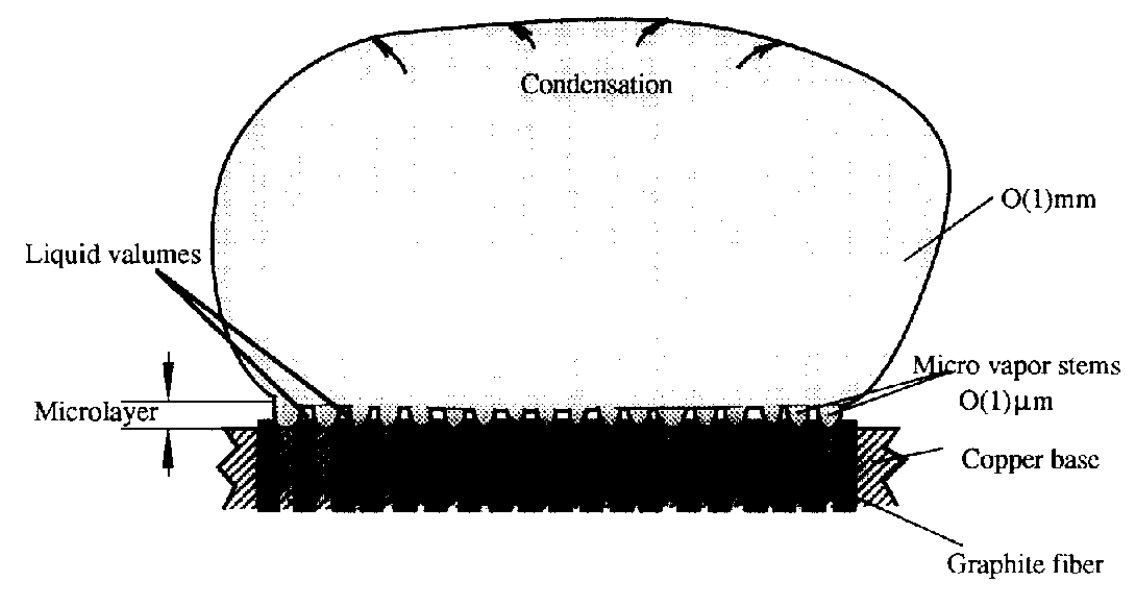

FIG. 4

\section{Single-Tier Configuration for Low Heat Flux Boiling on Composite Surface}

configuration which occurs in high heat-flux boiling on a composite surface. It should be noted that a large number of microbubbles are continually generated at the discretely distributed, active nucleation sites and ascend in columns into the macro liquid volumes in the macrolayer. Some of these microbubbles condense during their ascension, while others form long micro vapor stems, as seen in Fig. 5. One may conclude that the major contributors to the nucleate boiling performance are, again, the microbubbles in the fully-established nucleate boiling region at high heat fluxes up to the critical heat flux (CHF). The macro mushroom is nothing but a conglomerate of coalesced discrete bubbles.

In summary, the partial and fully-established nucleate boiling regions are characterized by the single- and double-tier configurations, respectively, as shown in Fig. 6.

\section{Estimation of Microlaver and Macrolaver Thicknesses}

Both the microlayer and macrolayer thicknesses are of great importance to nucleate boiling heat transfer performance. A variety of possible mechanisms of the macrolayer formation and quantitative data on the macrolayer thickness in the nucleate boiling on a plain surface have been reviewed by Sadasivan et al. [16]. $\Lambda$ rather large discrepancy exists in the results obtained by different investigators regarding surface roughness and wettability due to the use of different measuring techniques. However, the majority of the researchers agreed that the macrolayer thickness is of the order of hundreds of micrometer. The microconfiguration on the composite surface used in the present study is of a reasonably uniform size regarding the graphite fiber tips, which are rather uniformly distributed throughout the base material matrix, as shown in Fig. 2. Hence, the active nucleation sites which are formed by the spaces between the 

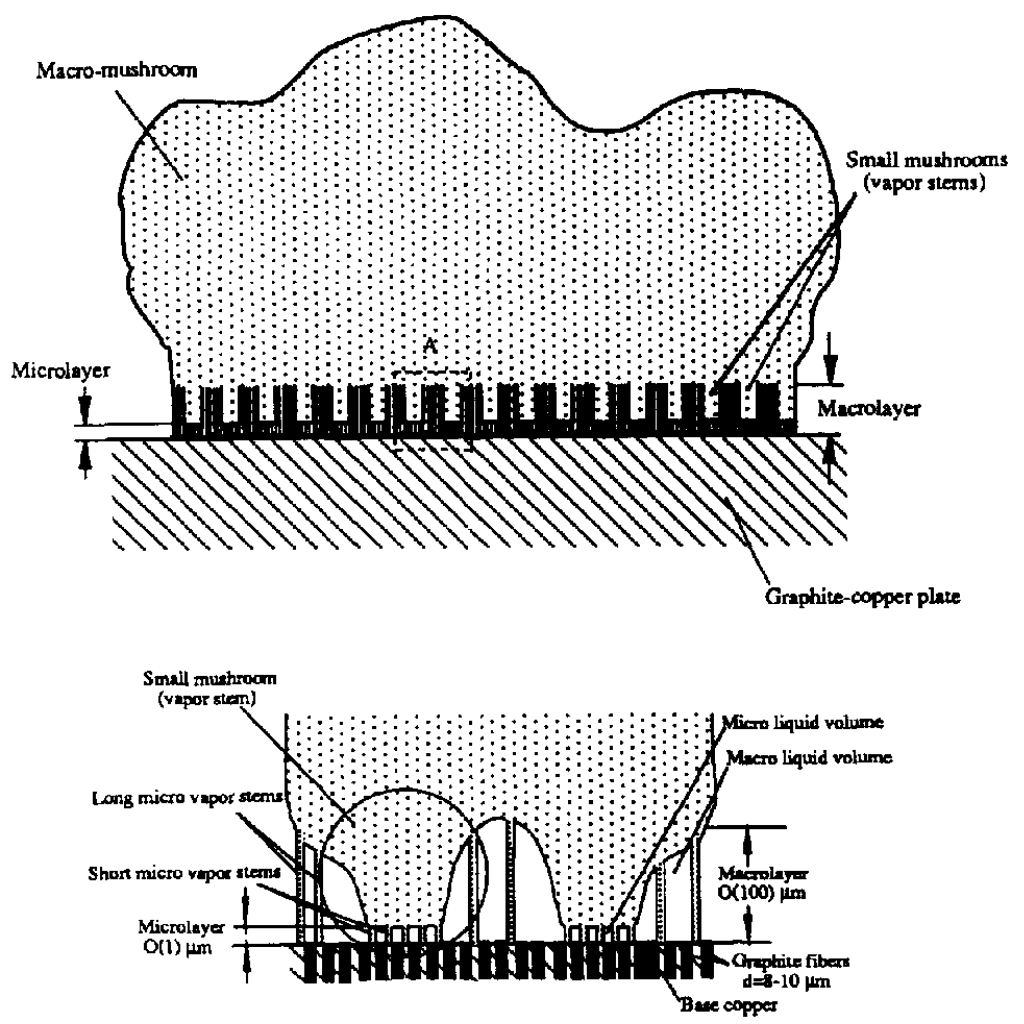

Detalls of $A$ area

FIG. 5

Two-Tier Configuration for High Heat Flux Boiling on Composite Surface

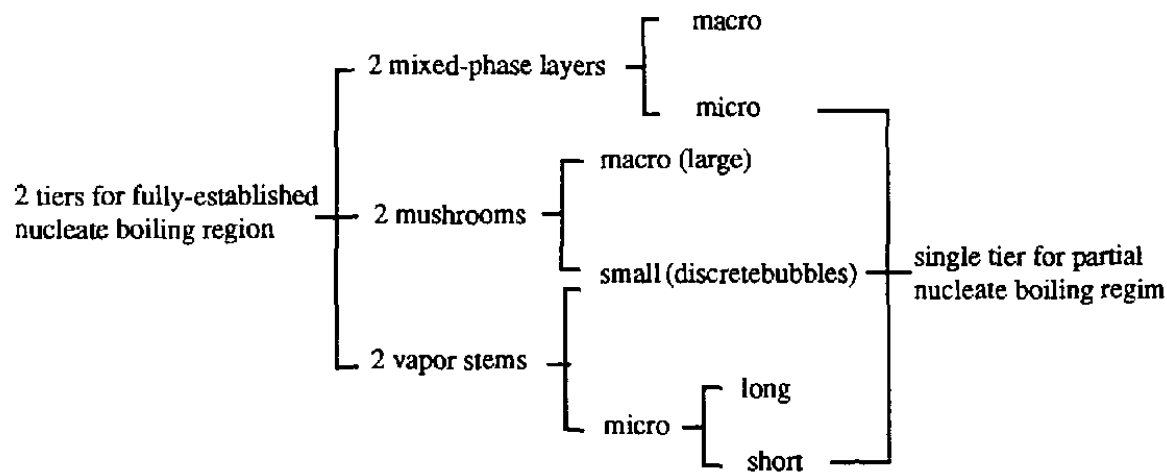

FIG. 6

Single- and Two- Tier Models for the Partial and Fully- Established Nucleate Boiling Regions 
fiber tips are of the same size and are uniformly distributed over the composite surface. It is postulated that each microbubble (with its base identified by a broken circle and its diametrical cross section by a solid circle) is situated in the middle of four neighboring fiber tips (identified by a dark circle), as shown in Fig. 7(a), having its surface near the base in point contact with the four tips, as depicted in Fig. 7(b). Here, d denotes the diameter of the graphite fibers; D, the diameter of microbubbles when they coalesce at their maximum cross section; $\mathrm{s}$, the diameter of the microbubble base; $\delta_{m}$, the height of the microbubble center from the base matrix surface; and $\beta$, the contact angle. Figure 7(c) illustrates the side view of the cross section in Fig. 7(a).

Let $\alpha$ be the area fraction of the fibers in the base material. The definition of $\alpha$ yields

$$
\alpha A=n \frac{\pi d^{2}}{4}
$$

where $A$ denotes the entire boiling surface area and $\mathrm{n}$ is the number of graphite fibers. When the microbubbles coalesce at their maximum cross section, as shown in Fig. 7(c), their diameter is related to the fiber diameter by

$$
D=\sqrt{\frac{\pi}{a}}\left(\frac{d}{2}\right)
$$

The plane of coalescence of microbubbles, $\delta_{\mathrm{m}}$, is regarded as the maximum thickness of

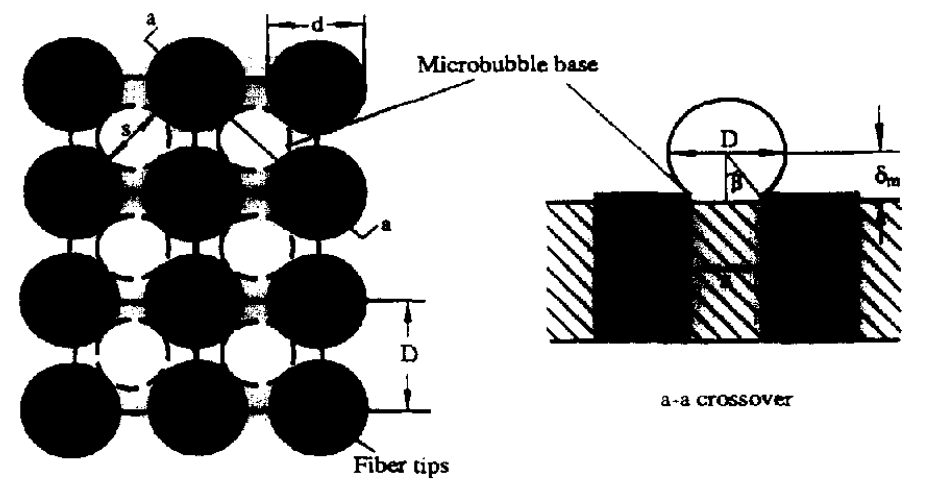

(a) top view of multiple microbubbles and fiber tips

(b) side view of a single microbubble on the composite surface

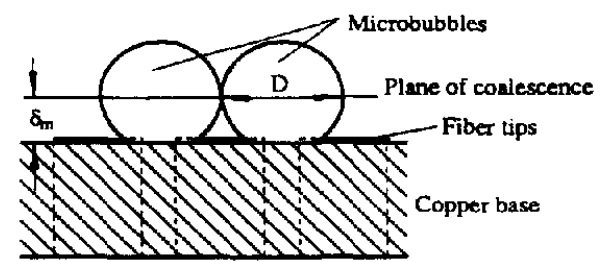

(c) side view of cross section

FIG. 7

Mechanism of Microlayer Formation on the Composite Surface 
the liquid film entrapped between the microbubbles. It is found to be

$$
\delta_{m}=\sqrt{\left(\frac{D}{2}\right)^{2}-\left(\frac{s}{2}\right)^{2}}=\frac{d}{2} \sqrt{\frac{\pi}{4 \alpha}-\left(\sqrt{\frac{\pi}{2 \alpha}}-1\right)^{2}}
$$

Using the method of calculating the macrolayer thickness [16], the volume of vapor below the plane of coalescence is

$$
V_{v}=\frac{1}{12} \pi D^{3}-\left[\frac{\pi}{24} D^{3}\left(2-3 \cos \beta+\cos ^{3} \beta\right)\right]=\frac{\pi}{8} D^{3}\left(\cos \beta-\frac{1}{3} \cos ^{3} \beta\right)
$$

The contact angle, $\beta$ is related to $\delta_{\mathrm{m}}$ by

$$
\cos \beta=\frac{2 \delta_{m}}{D}
$$

The volume of liquid trapped between the microbubbles is

$$
\begin{aligned}
V_{l} & =D^{2} \delta_{m}-\frac{\pi}{8} D^{3}\left(\cos \beta-\frac{1}{3} \cos ^{3} \beta\right) \\
& =D^{2} \delta_{m}-\frac{\pi}{4} D^{2} \delta_{m}+\frac{\pi}{3} \delta_{m}^{3}
\end{aligned}
$$

By idealizing the liquid volume as a column of constant cross-section, the microlayer thickness (equivalent to the liquid film thickness) is derived as

$$
\delta=\left(D^{2} \delta_{m}-\frac{\pi}{4} D^{2} \delta_{m}+\frac{\pi}{3} \delta_{m}^{3}\right) /\left[D^{2}-\pi\left(\frac{D^{2}}{4}-\delta_{m}^{2}\right)\right]
$$

For the area function of $50 \%$, combining Eqs. (2), (3) and (7) to eliminate D and $\delta_{\mathrm{m}}$, one obtains

$$
\delta=0.265 \mathrm{~d}
$$

The average fiber tip diameter of $9 \mu \mathrm{m}[6]$ and Eq. (8) predicts the microlayer thickness to be 2.4 $\mu \mathrm{m}$.

In the estimation of the macrolayer thickness, one uses the same contact angle for the discrete bubbles and the microbubbles. Then, it yields

$$
\beta=\arccos \frac{2 \delta_{m}}{D} \approx 38^{\circ}
$$

If the discrete bubbles which coalesce to form a vapor mass (macro mushroom) are of the same diameter $D_{m}$ and are uniformly distributed over the area underneath the vapor mass, one obtains the liquid volume being entrapped between the discrete bubbles as 


$$
V_{l, m}=\frac{1}{2} D_{m}^{2} \cos \beta-\frac{\pi}{8} D_{m}^{3}\left(\cos \beta-\frac{1}{3} \cos ^{3} \beta\right)
$$

By idealizing this liquid volume to be a column of constant cross section, the macrolayer thickness (equivalent to the thickness of the liquid layer entrapped between the discrete bubbles) is obtained as

$$
\begin{aligned}
\Delta & =\left[\frac{1}{2} D_{m}{ }^{3} \cos \beta-\frac{\pi}{8} D_{m}{ }^{3}\left(\cos \beta-\frac{1}{3} \cos ^{3} \beta\right)\right] /\left(D_{m}{ }^{2}-\frac{\pi}{4} D_{m}{ }^{2} \sin ^{2} \beta\right) \\
& =\frac{\pi}{2} D_{m} \cos \beta\left[1-\frac{\pi}{4}\left(1-\frac{1}{3} \cos ^{2} \beta\right)\right] /\left(1-\frac{\pi}{4} \sin ^{2} \beta\right)
\end{aligned}
$$

Referring to the boiling of water on plain surfaces [4] and that of Freon 113 on the microconfigured surfaces [6], the diameter of discrete bubbles $D_{m}$ is estimated to be approximately $1 \mathrm{~mm}$. Then, Eq. (11) predicts the macrolayer thickness to be approximately 210 $\mu \mathrm{m}$.

\section{Discussion}

The model can be extended to nucleate boiling on plain surfaces on which numerous micro cavities may function as active nucleation sites. The distribution of these cavities is random but its pattern can be classified into three categories: uniformly distributed cavity site, $\mathrm{A}$, a densely populated cavity site, B, and a scarcely populated cavity site, C, as depicted in Fig. 8 (these sites are not at their actual locations).

\section{(1) Low heat flux region}

In the case of the partial nucleate boiling, a discrete bubble is suspended on a mixedphase microlayer. There exist in the microlayer (i) uniformly-distributed micro vapor stems on the area $A$, (ii) micro vapor slugs on the area $B$, and (iii) individual micro vapor stems on the area $C$. Accordingly, three mechanisms of heat transfer take place simultaneously within the microlayer: (i) latent heat transport in the micro vapor stems $\mathrm{q}_{\mathrm{A}}$ in the area $\mathrm{A}$, (ii) conductive heat transfer in the micro vapor slugs $\mathrm{q}_{B}$ in the area $B$, and (iii) conductive heat transfer in the micro liquid film $\mathrm{q}_{C}$ in the area $C$. In general, the magnitudes of both $\mathrm{q}_{B}$ and $\mathrm{q}_{C}$ are smaller than that of $\mathrm{q}_{\mathrm{A}}$. Discrete bubbles are preferably formed on densely-populated cavity site areas with their base centered in the region of micro vapor slugs.

\section{(2) High heat flux region}

For nucleate boiling on plain surfaces, the macro mushroom generated in the high heat flux region is similar in construction to the discrete bubble mentioned above. Therefore, its boiling heat transfer mechanisms follow those found in the low heat flux region. 


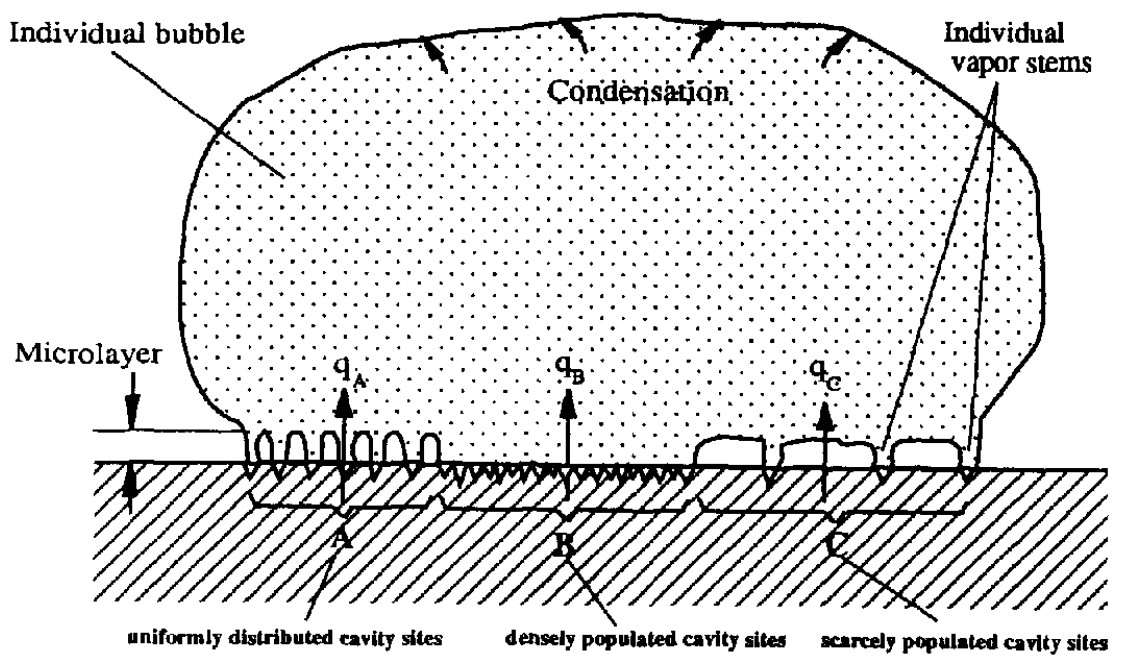

FIG. 8

Discrete Bubble in Partial Nucleate Boiling Region

In contrast, a graphite-copper composite surface has the following enhancement features:

1. It has more active nucleation sites which are provided by a planned arrangement of graphite fibers.

2. The latent heat transport in the micro vapor stems is the dominant heat transfer mode.

The contribution of conductive heat transfer through both of the micro liquid films and micro vapor slugs is very minor, due to the uniform distribution of active nucleation sites.

3. There is more intense heat from the graphite fiber tips directly into the microbubbles, especially when the tips become hot spots.

The present model has revealed that the primary contributors of the boiling heat transfer are the microbubbles in the microlayers and heat conduction in the micro liquid films. Accordingly, the boiling heat flux can be written as

$$
q=w_{1} q_{b}+w_{2} q_{1}
$$

Here, $q_{b}$ and $q_{l}$ are the heat fluxes transported by the microbubbles and micro liquid film, respectively, and $w_{1}$ and $w_{2}$ are their weighting fractions. They can be expressed as

$$
\begin{aligned}
& q_{b}=f\left(\Delta t_{w}\right) n\left(\Delta t_{w}\right) \frac{1}{6} \pi d^{3} \rho_{v} h_{f 8} \\
& q_{l}=k_{l} \Delta t_{w} / \delta_{m}
\end{aligned}
$$

in which $f$ is the microbubble departure frequency; $n$ is the number of active nucleation sites; $\rho_{\mathrm{v}}$ is the vapor density; $h_{f_{8}}$ is the latent heat of vaporization; $k_{l}$ is the liquid conductivity; and $\Delta \mathrm{t}_{\mathrm{w}}$ is the temperature difference between the heating surface and the saturation liquid. Both $f$ 
and $n$ are functions of $\Delta t_{w}$. In comparison with these two heat transfer components, the heat of evaporation from the portion of the bubble surface that is immersed in the thermal layer is negligible.

\section{Conclusions}

The two-tier model has been developed to elucidate the mechanisms of nucleate boiling heat transfer on the microconfigured composite surfaces. Both the microlayer and macrolayer thicknesses can be evaluated using this model. The model can be extended to explain the nucleate pool boiling mechanisms on plain surfaces. The enhanced measurements of nucleate boiling heat transfer on the composite surface have been explained through a comparison of the two-tier constructions on the composite and plain surfaces.

\section{Nomenclature}

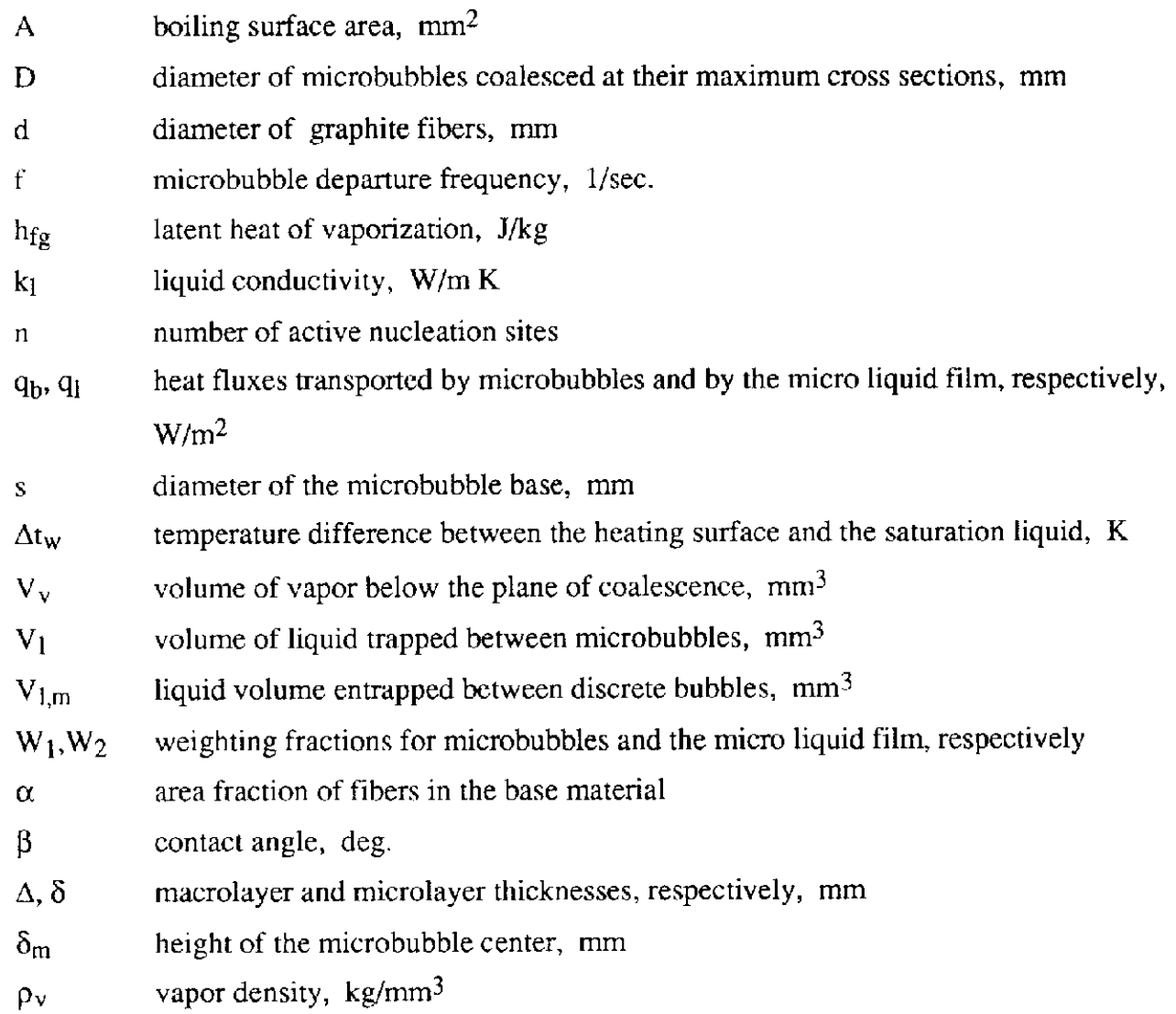

\section{References}

1. Thome, J. R., Engineering Foundation Conference on Pool and External Flow Boiling, Santa Barbara, (1992).

2. Gebhart, B. and Wright, N., Int. Comm. Heat Mass Transfer, 15, No. 2, 141 (1988) 
3. France, A. M., M. S. Thesis, University of Pennsylvania, Philadelphia, Pennsylvania (1991)

4. Miller, W. J., M. S. Thesis, University of Pennsylvania, Philadelphia, Pennsylvania (1991)

5. Wright, N. T. and Gebhart, B., Engineering Foundation Conference on Pool and External Flow Boiling, Santa, Barbara (1992)

6. Yang, W. -J., Takizawa, H. and Vrable, D. L., Int. J. Heat Mass Transfer, 34, No. 11, 2751 (1991)

7. Gaertner, R. F., J. Heat Transfer, $\underline{87}, 17$ (1965)

8. Kirby, D. B. and Westwater, J. W., Chemical Engineering Progress Symposium Series, $\underline{61}$, No. $51,238(1965)$

9. Ishigai, S. and Kuno, T., Bulletin J. S. M. E., 9, 361 (1966)

10. Katto, Y. and Yokoya, S., Int. J. Heat Mass Transfer, 11, 993 (1968)

11. Iida, Y. and Kobayasi, K., Bulletin J. S. M. E., 12 , 283 (1969)

12. Bhat, A. M., Saini, J. S. and Prakash, R., Int. J. Heat Mass Transfer, 29, 1951 (1986)

13. Yu, C. L. and Mesler, R. B., Int. J. Heat Mass Transfer, 20, 827 (1977)

14. Fujita, Y., Engineering Foundation Conference on Pool and External Flow Boiling, Santa Barbara, CA, March 22-27, 1992

15. Yang, G.W., Yang, W. -J. and Zhang, N., to appear in Int. Comm. Heat Mass Transfer

16. Sadasivan, P., Chappidi, P. R., Unal, C. and Nelson, R. A., Engineering Foundation Conference on Pool and External Flow Boiling, Santa Barbara, CA, March 22-27, 1992

Received July 27, 1992 